\title{
Erratum to: Resonance assignments of cohesin and dockerin domains from Clostridium acetobutylicum ATCC824
}

Zhenling Cui $\cdot$ Yifei Li $\cdot$ Yan Xiao
Yingang Feng $\cdot$ Qiu Cui

Published online: 29 April 2012

(C) The Author(s) 2012

\section{Erratum to: Biomol NMR Assign}

DOI 10.1007/s12104-012-9381-2

Unfortunately, the fifth author name was misspelled in the original publication. The correct name should read as "Qiu
Cui." Additionally, the author would like to correct the affiliation. The correct information is given in this article.

The online version of the original article can be found under doi:10.1007/s12104-012-9381-2.

Z. Cui · Y. Li ( $₫) \cdot$ Y. Xiao · Y. Feng · Q. Cui $(\bowtie)$

Shandong Provincial Key Laboratory of Energy Genetics, Key Laboratory of Biofuels, Qingdao Institute of Bioenergy and Bioprocess Technology, Chinese Academy of Sciences, No. 189 Songling Road, Qingdao 266101, China

e-mail: liyf@qibebt.ac.cn

Q. Cui

e-mail: cuiqiu@qibebt.ac.cn 